\title{
OCORRÊNCIA DE ÁCAROS EM AMOSTRAS DE POEIRA DE SOFÁS DE RESIDÊNCIAS UNIVERSITÁRIAS DE UBERABA, MG
}

\author{
MIRA, Camila da Cruz ${ }^{1}$ \\ PEREIRA, Fernando Lourenço ${ }^{2}$
}

\begin{abstract}
RESUMO: As reações alérgicas decorrentes da exposição à alérgenos ambientais são responsáveis pela ocorrência de doenças como a asma, rinite alérgica, rinoconjuntivite e dermatite atópica. Alérgenos de ácaros da poeira domiciliar são importantes na etiologia das doenças alérgicas. Portanto, o conhecimento da acarofauna em poeira domiciliar poderá ser uma medida adicional para o controle de ácaros no ambiente domiciliar. O objetivo deste estudo foi identificar as espécies de ácaros mais encontradas em poeira domiciliar de residências universitárias da cidade de Uberaba/MG. Foram coletadas aleatoriamente 10 amostras de poeira domiciliar em sofás. Foi utilizado o método de suspensão de Fernandez-Caldas (1997) para preparo das amostras e posterior identificação acarológica. A família mais frequente foi Pyroglyphidae com as espécies Dermatophagoides pteronyssinus (40,93\%), Dermatophagoides farinae (36,92\%), Dermatophagoides sp. (17,30\%), Dermatophagoides deanei e Sturnophagoides brasiliensis $(0,21 \%$ ambas). A família Glycyphagidae apareceu com Blomia tropicalis $(0,84 \%)$. A família Sarcoptidae apareceu com Sarcoptes scabiei $(0,21 \%)$, incomum em poeira domiciliar por se tratar de ácaro parasita. A literatura aponta as espécies Dermatophagoides pteronyssinus, Dermatophagoides farinae e Blomia tropicalis como alergênicas. Dessa forma, foi possível orientar os moradores das residências universitárias, sobre os cuidados, higienização dos sofás e outros microambientes adequados para a nidificação e proliferação de ácaros.
\end{abstract}

Palavras-chave: Ácaros. Poeira domiciliar. Aeroalérgenos. Doenças respiratórias alérgicas.

SUMMARY: Allergic reactions, due to exposure to environmental allergens are responsible for the occurrence of diseases such as asthma, allergic rhinitis, rhinoconjunctivitis and atopic dermatitis. Dust mite allergens are important in the etiology of allergic diseases. Therefore, the knowledge of the house dust mite may be an additional measure for the control of house dust mites. The objective of this study was to identify the most common house dust mite species found in university residences in the city of Uberaba / MG. Samples of house dust on sofas were randomly collected. The suspension method of Fernandez-Caldas (1997) was used to prepare the samples and subsequent acarological identification. The most frequent family was Pyroglyphidae with the species Dermatophagoides pteronyssinus (40,93\%), Dermatophagoides farinae (36,92\%), Dermatophagoides sp. (17.30\%), Dermatophagoides deanei and Sturnophagoides brasiliensis ( $0.21 \%$ both). The Glycyphagidae family appeared with Blomia tropicalis $(0.84 \%)$. The Sarcoptidae family appeared with Sarcoptes scabiei $(0.21 \%)$, which is unusual in house dust because it is a parasitic mite. The literature shows the species Dermatophagoides pteronyssinus, Dermatophagoides farinae and Blomia tropicalis as allergens. In this way, it was possible to guide the residents of the university residences on the care, hygiene of the sofas and other microenvironments suitable for nesting and proliferation of mites.

Keywords: Mites. House dust. Aeroallergens. Respiratory allergic diseases.

\section{INTRODUÇÃO}

Alergia é o termo utilizado para caracterizar a hipersensibilidade de um organismo frente a um alérgeno. Essa hipersensibilidade pode ser classificada como hipersensibilidade do tipo I ou imediata quando a resposta imune é mediada pela imunoglobulina $\mathrm{E}$, que se liga a receptores na superfície de mastócitos (ABBAS, LICHTMAN e PILLAI, 2014).

As alergias são desencadeadas por fatores genéticos e ambientais nos quais indivíduos

\footnotetext{
${ }^{1}$ Graduada em Ciências Biológicas pela Universidade Federal do Triângulo Mineiro

${ }^{2}$ Docente do Instituto de Ciências Exatas, Naturais e Educação da Universidade Federal do Triângulo Mineiro
} 
predispostos geneticamente produzem anticorpos IgE frente a proteínas inócuas do ambiente (alérgenos), resultando em reações inflamatórias nas vias aéreas e/ou pele e mucosa intestinal. Essas reações alérgicas, decorrentes da exposição à alérgenos ambientais, são responsáveis pela ocorrência de doenças como a asma, rinite alérgica, rinoconjuntivite e dermatite atópica na população (SEGUNDO et al. 2009).

Os alérgenos podem apresentar origem glicoproteica, proteica ou como carboidratos isolados (JOHANSSON et al. 2004). Quando dispersos no ambiente e transportados pelo ar, os alérgenos são denominados aeroalérgenos e estão associados a diferentes organismos pertencentes ao Reino Plantae, Fungi e Animalia. Devido a essa variedade, são divididos em domiciliares quando estão associados às partículas derivadas de restos corporais e fezes de ácaros, baratas e outros animais e extradomiciliares quando estão associados a grãos de pólen e gramíneas (GALLY e LANTZ, 1999).

Os aeroalérgenos são os principais agentes etiológicos das doenças alérgicas respiratórias. A sensibilização e os sintomas frente a essa exposição podem se intensificar de acordo com a frequência em que ela ocorre e a faixa etária do indivíduo. Com isso, nota-se um aumento do predomínio de doenças alérgicas respiratórias na população global, principalmente em crianças, devido ao estilo de vida atual no qual as pessoas permanecem longos períodos em ambientes fechados com pouca circulação de ar. Outros fatores que podem levar a esse aumento são as alterações ambientais como mudanças climáticas bruscas e poluição, tanto no ambiente domiciliar quanto no extradomiciliar (SOUZA e ROSARIO FILHO, 2012).

Dentre os alérgenos importantes na etiologia das doenças alérgicas destacam-se àqueles provenientes de ácaros da poeira domiciliar. Um estudo realizado em Curitiba constatou a relação entre rinite alérgica e ácaros por meio de testes cutâneos positivos para alérgenos da espécie de ácaro Dermatophagoides pteronyssinus. Os dados obtidos demonstraram que há recorrência de rinite alérgica em $12,2 \%$ de crianças e adolescentes e $25,4 \%$ dos adultos que participaram do estudo (ESTEVES, ROSARIO e ZAVADINIAK, 2000). Portanto, estudos que fomentem a avaliação da acarofauna em ambientes domiciliares e extradomiciliares são importantes para medidas de controle desses animais, uma vez que visa a redução de aeroalérgenos provenientes dos corpos e fezes desses artrópodes.

Os ácaros são artrópodes microscópicos que se nidificam na poeira e em condições adequadas do micro e macroambiente se reproduzem com facilidade (SOUZA, 2013). Geralmente, apresentam característica de ciclo de vida com metamorfose completa, pois durante o seu desenvolvimento passam pelos estágios de ovo, larva, protoninfa, tritoninfa e adulto. Cada espécie possui as suas particularidades, dessa forma, padrões de características morfológicas e tolerância a pequenas variações na temperatura, umidade realtiva do ar e disponibilidade de alimento variam de acordo com a espécie (PIKE et al. 2005). Em aspectos gerais, temperaturas entre 20 e $30^{\circ} \mathrm{C}$ e umidade relativa do ar entre 50 e $80 \%$ propiciam condições adequadas para a proliferação de ácaros (MIYAMOTO et al. 1995).

Em residências é possível encontrar muitos microambientes adequados para a sobrevivência e desenvolvimentos de ácaros, como sofás, colchões, almofadas e tapetes. Nesses locais, há o acúmulo de poeira, condições de temperatura, umidade e nutrição favoráveis para o desenvolvimento dos ácaros devido à transpiração e descamação da pele de pessoas e animais. Além do microambiente ser adequado é necessária, também, a adequação do macroambiente, pois este influencia fortemente na dinâmica da população (CAMPOS; LIPORACI; TERRA, 2010).

A identificação de alérgenos presentes na poeira domiciliar é importante, pois as constantes modificações de temperatura e umidade do micro e macroambiente influenciam na fauna alergênica. Além disso, essa identificação permite melhor intervenção com tratamento adequado e orientações cabíveis

( SOUZA; ROSARIO FILHO, 2012). Um estudo de Terra e colaboradores (2004) apontou Dermatophagoides pteronyssinus e Dermatophagoides farinae como as espécies de ácaros mais encontradas em poeira domiciliar da cidade de Uberaba/MG. 
Souza (2013) verificou a predominância de aeroalérgenos nas residências de pacientes diagnosticados com asma e/ou rinite alérgica em Curitiba/PR e relacionou os dados com a exposição, sensibilização e manifestações clínicas desses pacientes. Nesse trabalho concluiu-se que a espécie $D$. pteronyssinus é mais frequente nas residências, seguida pelas espécies D. farinae e Blomia tropicalis. Em residências com presença de animal doméstico foram encontrados maiores níveis de concentração alergênica de ácaros.

Alérgenos de ácaros podem representar um problema significante em indivíduos sensibilizados, especialmente aqueles que tenham frequente contato com os locais onde os ácaros são encontrados. Assim, o conhecimento da acarofauna em poeira domiciliar poderá ser uma medida adicional para prevenir sintomas de doenças respiratórias como asma e rinite alérgica.

O presente estudo teve como objetivo identificar as espécies de ácaros mais encontradas em poeira domiciliar de residências universitárias da cidade de Uberaba/MG e determinar a frequência das populações de ácaros encontradas.

\section{MATERIAL E MÉTODO}

A pesquisa foi realizada na cidade de Uberaba (MG) durante a primeira quinzena de fevereiro e primeira quinzena de abril de 2017. A média de temperatura foi de $29,66^{\circ} \mathrm{C}$ e a média de umidade relativa do ar foi de 70\%, no momento das coletas. Um representante da residência assinou o termo de consentimento autorizando a coleta da amostra. Foram coletadas, aleatoriamente, dez amostras de poeira de sofás de residências universitárias sendo cinco localizadas no bairro Nossa Senhora da Abadia, três no bairro Universitário e duas no bairro Olinda. As amostras foram coletadas de acordo com os seguintes critérios: (1) o sofá deveria ser revestido por tecido, (2) deveria pertencer a uma residência universitária, e (3) deveria estar em uso pelos universitários.

Para a coleta foi utilizado um aspirador de pó (Philco PH1390 MAXX 1200 W) adaptado com filtro de papel (marca Melitta) para a retenção da poeira coletada (Figura 1.A,B,C e 2). Cada aspiração de cada sofá durou 15 minutos, sendo um sofá por residência universitária. Em seguida, as amostras foram codificadas e armazenadas em geladeira a $4^{\circ} \mathrm{C}$ até o preparo da técnica para a identificação da fauna acarina. No momento da coleta foi observada a presença ou ausência de animais domésticos (cães e gatos). No fim de cada coleta, o tubo prolongador, a mangueira flexível, o acessório de aspiração e o saco coletor de poeira do aspirador, foram higienizados com água corrente, detergente, álcool 70\% e secagem com papel absorvente para não contaminar as outras amostras.

Para a identificação dos ácaros, as amostras foram peneiradas para diminuir a presença de pelos e fibras de tecido. Em seguida, foi utilizado o método de suspensão de Fernandez-Caldas (1997). Esse método consiste na adição de $50 \mathrm{mg}$ de poeira coletada em $10 \mathrm{~mL}$ de solução salina saturada e $20 \mathrm{~mL}$ de álcool-éter na proporção 1:1. Em seguida o material passou por um período de incubação em temperatura ambiente por 5 minutos seguido de leve agitação e disposição da mistura em duas placas de Petri. As misturas foram analisadas em microscópio estereoscópio em aumento de 40X. Os ácaros encontrados foram depositados em lâmina juntamente com 2 gotas de meio de preparação de Hoyer (4 $\mathrm{mL}$ de água destilada, $3 \mathrm{~mL}$ de goma arábica, $20 \mathrm{~g}$ de hidrato de cloral e $2 \mathrm{~mL}$ de glicerina, adicionados nesta ordem). As lâminas foram cobertas por lamínulas e vedadas por esmalte incolor. Em seguida, foram armazenadas em estufa a $50^{\circ} \mathrm{C}$ por 48 horas para a clarificação do material. 
Figura 1 - A - Saco coletor de poeira do aspirador sem adaptação com filtro de papel. B e C Saco coletor de poeira adaptado com filtro de papel em seu interior.
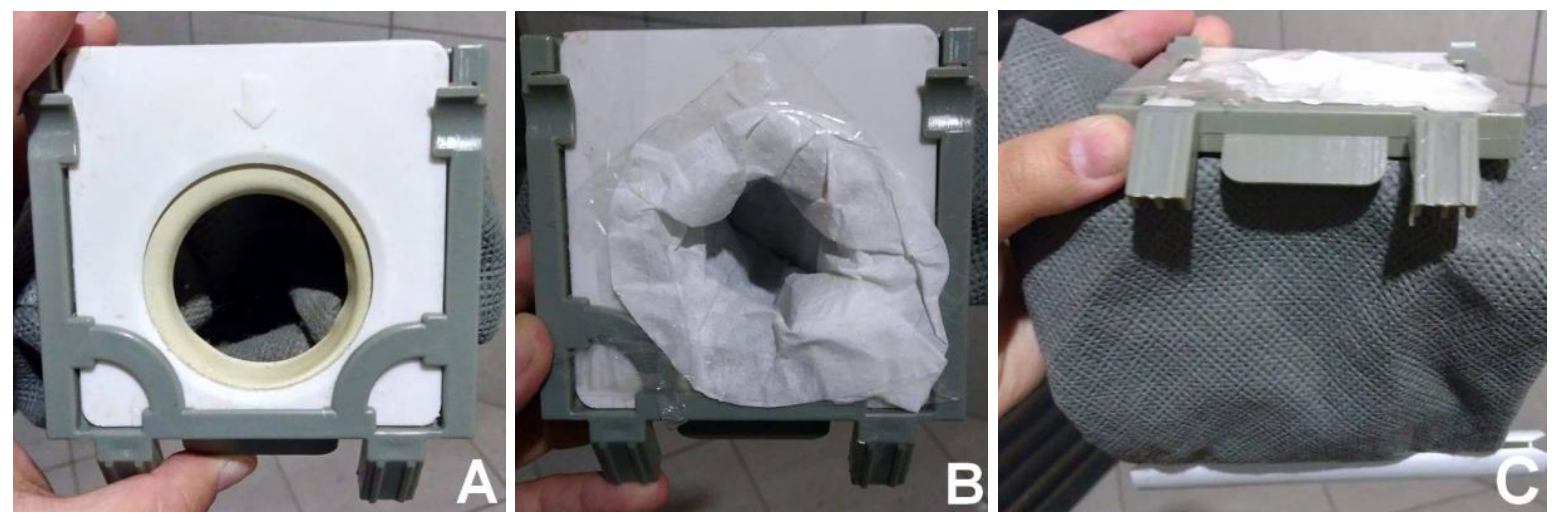

Fonte: Arquivo pessoal de Camila da Cruz Mira.

Figura 2 - Poeira retida no filtro de papel após a coleta

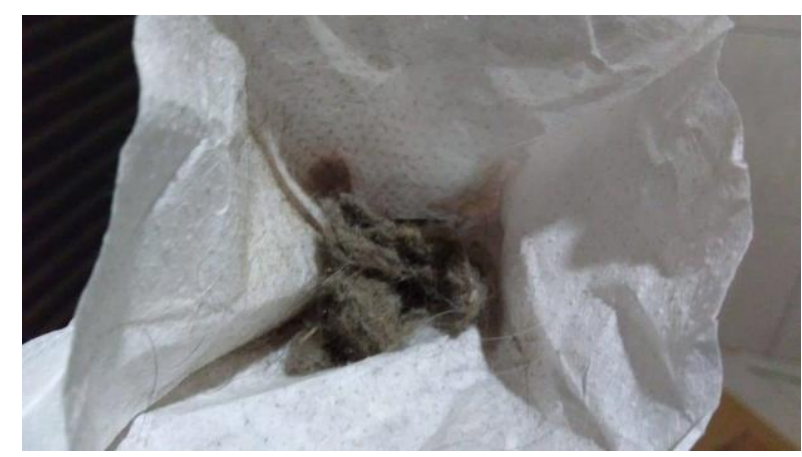

Fonte: Arquivo pessoal de Camila da Cruz Mira.

Após a clarificação do material, as lâminas foram observadas em microscópio de luz nos aumentos de 40X, 100X, 400X e 1000X (quando necessário). As espécies foram identificadas com base nos estudos taxonômicos de Galvão e Guitton (1986a , 1986b , 1989) e Flechtmann (1973). Foi realizada a classificação dos ácaros em gênero e, sempre que possível, em espécie. Também foram subclassificados com relação ao sexo e estágio de desenvolvimento, como larva e ninfas.

A análise estatística foi realizada através da determinação da frequência absoluta $(\mathrm{N})$ e frequência relativa $(\%)$ das populações.

\section{RESULTADO E DISCUSSÃO}

Foram encontrados ácaros em todas (100\%) as amostras coletadas. Através da análise acarológica, foram identificados representantes de três famílias: Glycyphagidae, Sarcoptidae e Pyroglyphidae. A família Pyroglyphidae foi a mais frequente encontrada entre as amostras analisadas. Esse estudo está de acordo com um levantamento bibliográfico realizado por Binotti (2001) que constatou que as famílias de ácaros mais encontradas em poeira domiciliar no Brasil são: Pyroglyphidae, Glycyphagidae, Cheyletidae e Acaridae.

Das amostras processadas e analisadas, três apresentaram quantidade igual ou superior a 80 ácaros, três apresentaram entre 30 e 50 ácaros e em quatro foram quantificados abaixo de 30 ácaros (Tabela1). Os dados obtidos neste trabalho não demonstraram relação entre a quantidade de 
moradores na residência, a presença ou ausência de animais domésticos e a quantidade de ácaros identificados em cada amostra.

Tabela 1 - Relação entre quantidade de ácaros encontrados nas amostras de poeira domiciliar coletadas, quantidade de pessoas residentes e presença de animais domésticos nas residências universitárias de Uberaba/MG.

\begin{tabular}{lccc}
\hline $\begin{array}{c}\text { Código da } \\
\text { Amostra }\end{array}$ & $\begin{array}{c}\text { Número de } \\
\text { ácaros/amostra }\end{array}$ & $\begin{array}{c}\text { Presença de animais nas } \\
\text { residências (N)* }\end{array}$ & $\begin{array}{c}\text { Número de residentes nas } \\
\text { residências universitárias }\end{array}$ \\
\hline S01 & 112 & Sim (1) & 7 \\
S02 & 13 & Não & 3 \\
S03 & 85 & Sim (1) & 5 \\
S04 & 56 & Sim (1) & 4 \\
S05 & 28 & Sim (1) & 4 \\
S06 & 80 & Não & 6 \\
S07 & 20 & Sim (2) & 8 \\
S08 & 33 & Não & 5 \\
S09 & 32 & Não & 4 \\
S10 & 15 & Não & \\
TOTAL & 474 & & \\
\hline
\end{tabular}

*onde $\mathrm{N}$ é a quantidade de animais domésticos presentes na residência universitária.

As espécies de ácaros em ordem decrescente de frequência foram: Dermatophagoides pteronyssinus (40,93\%), Dermatophagoides farinae (36,92\%), Dermatophagoides sp. (17,30\%), Blomia tropicalis (0,84\%), Dermatophagoides deanei, Sturnophagoides brasiliensis e Sarcoptes scabiei (0,21\%). Também foram encontrados larvas $(1,48 \%)$, protoninfas $(0,42 \%)$ e tritoninfas $(1,27 \%)$. Não foi observada a presença de ovos nas amostras analisadas. Um ácaro não pôde ser devidamente identificado por não apresentar condições de preservação e clarificação adequadas (Tabela 2).

Tabela 2 - Taxonomia e frequência de ácaros encontrados em sofás de residências universitárias em que foram coletadas as amostras de poeira domiciliar na cidade de Uberaba/MG.

\begin{tabular}{lc}
\hline \multicolumn{1}{c}{ Taxonomia } & Frequência N(\%)* \\
\cline { 1 - 2 } Família Glycyphagidae & \\
Blomia tropicalis & $4(0,84)$ \\
\cline { 1 - 2 } $\begin{array}{l}\text { Família Sarcoptidae } \\
\text { Sarcoptes scabiei }\end{array}$ & $1(0,21)$ \\
\cline { 1 - 2 } Família Pyroglyphidae & \\
Dermatophagoides deanei & $1(0,21)$ \\
Dermatophagoides farinae & $175(36,92)$ \\
Dermatophagoides pteronyssinus & $194(40,93)$ \\
Dermatophagoides sp. & $82(17,30)$ \\
Sturnophagoides brasiliensis & $1(0,21)$ \\
\hline Larvas & $7(1,48)$ \\
Protoninfas & $2(0,42)$ \\
Tritoninfas & $6(1,27)$ \\
Não identificado & $1(0,21)$ \\
\hline TOTAL & $474(100)$ \\
*Frequência absoluta (N) e frequência relativa (\%) de ácaros para amostras positivas.
\end{tabular}

D. pteronyssinus apresentou maior frequência (40,93\%), sendo sua maioria fêmea $(59,79 \%)$ (Tabela 3). Esses resultados estão de acordo com os dados apresentados por Terra (2004) que avaliou a acarofauna em residências de famílias da cidade de Uberaba/MG. Estudos realizados por Binotti (2002, 
2003) na cidade de Campinas/SP e um estudo realizado por Panigalli (2014) na biblioteca universitária central da Universidade do Oeste de Santa Catarina (Unoesc) de Xanxerê também relataram $D$. pteronyssinus como espécie mais frequente nas amostras.

Em seguida, encontrou-se com mais frequência a espécie D. farinae (36,92\%), sendo sua maioria macho (58,86\%). Diferentemente de um estudo realizado por Campos (2010) que demonstra a predominância de ácaros da espécie $D$. farinae na cidade de Ituverava/SP.

Tanto D. pteronyssinus e D. farinae são espécies presentes em regiões tropicais e subtropicais. As populações estão condicionadas a diversos fatores para que possam se desenvolver e sobreviver como temperatura e umidade relativa do ar que devem estar adequadas às necessidades da espécie. Dessa forma, as condições de temperatura, umidade relativa do ar e disponibilidade de recursos como alimento das amostras analisadas na cidade de Uberaba favorecem o desenvolvimento de ácaros da espécie $D$. pteronyssinus. Explica-se, então a diferença de dados obtidos entre tais estudos (CAMPOS, 2010). O estudo realizado por Campos apresentou a umidade relativa do ar média de $62 \%$ enquanto que na cidade de Uberaba a média foi de $70 \%$, essa variação pode interferir na dinâmica da população de $D$. farinae.

Tabela 3 - Taxonomia, classificação quanto ao sexo e frequência de ácaros encontrados em sofás das residências universitárias em que foram coletadas as amostras de poeira domiciliar na cidade de Uberaba/MG.

\begin{tabular}{|c|c|c|}
\hline \multirow{2}{*}{ Taxonomia } & \multicolumn{2}{|c|}{$\operatorname{Sexo} N(\%)^{*}$} \\
\hline & 우 & $0^{\lambda}$ \\
\hline $\begin{array}{l}\text { Família Glycyphagidae } \\
\text { Blomia tropicalis }\end{array}$ & $3(75)$ & $1(25)$ \\
\hline Família Pyroglyphidae & & \\
\hline Dermatophagoides deanei & $1(100)$ & $0(0,00)$ \\
\hline Dermatophagoides farinae & $72(41,14)$ & $103(58,86)$ \\
\hline Dermatophagoides pteronyssinus & $116(59,79)$ & $78(40,21)$ \\
\hline Sturnophagoides brasiliensis & $1(100)$ & $0(0,00)$ \\
\hline TOTAL & 193 & 182 \\
\hline
\end{tabular}

*Frequência absoluta $(\mathrm{N})$ e frequência relativa (\%) de ácaros para amostras positivas.

A espécie Blomia tropicalis foi encontrada em menor frequência, esse resultado corrobora com o trabalho de Terra (2004), realizado na mesma cidade do presente estudo. Essa espécie representa grande importância médica para pacientes asmáticos, por apresentar alérgenos que ao serem reconhecidos pela IgE desencadeiam uma resposta alérgica nas pessoas sensibilizados (ALMEIDA, 2010; JESUS, 2006).

Entre as espécies encontradas com baixa frequência encontra-se Sarcoptes scabiei $(0,21 \%$ das espécies identificadas). Incomum em poeira domiciliar, pois trata-se de um ácaro parasita responsável pela sarna sacóptica em animais e sarna humana ou escabiose em seres humanos. Essa doença é causada por diferentes variedades de Sarcoptes scabiei que são denominadas de acordo com o hospedeiro que parasitam. O ácaro localiza-se na epiderme, sob a camada córnea e produz lesões na pele que facilitam contaminações secundárias por microorganismos. O contágio ocorre através da migração da fêmea presente em um indivíduo doente para um indivíduo sadio (FLECHTMANN, 1973). Este é um problema de saúde de escala mundial que demanda o diagnóstico e tratamento dos doentes de forma adequada e medidas profiláticas destinadas às comunidades mais afetadas (FARIA, 2011; RODRIGUES, 2014).

Foi constatado que os moradores da residência onde foi coletada a amostra positiva para Sarcoptes scabiei, haviam adotado recentemente um cachorro. Foi realizada uma busca e constatadas pequenas lesões cutâneas no animal, que até o momento, não haviam sido percebidas. O fato de o animal ter livre acesso a todos ambientes da casa, inclusive o sofá, explicam a presença de tal espécie na amostra. 
Dermatophagoides deanei também apresentou frequência relativa de 0,21\%. Essa espécie foi descrita pela primeira vez por Galvão e Guitton (1986) em amostras provenientes de poeira domiciliar de seis capitais brasileiras: São Luis, Fortaleza, Natal, Maceió, Belo Horizonte e Rio de Janeiro.

Sturnophagoides brasiliensis (0,21\%) foi descrita por Fain (1967), através de uma amostra coletada de poeira em uma residência na cidade Tejipio, localizada próxima a Recife, em 11 de julho de 1966. Além do Brasil foram relatadas recorrência dessa espécie na Espanha, Malásia, Singapura e Indonésia (COLLOFF, 2009).

Tanto a espécie Dermatophagoides deanei e Sturnophagoides brasiliensis não são muito descritas na literatura por se tratar de espécies pouco recorrentes. Dessa forma, não foi possível verificar se essas espécies apresentam alérgenos que estão relacionados às doenças alérgicas respiratórias.

A baixa frequência de larvas $(1,48 \%)$, protoninfas $(0,42 \%)$, tritoninfas $(1,27 \%)$ e ausência de ovos indica que, provavelmente, os indivíduos da população não se encontravam em período reprodutivo no momento em que as amostras foram coletadas.

\section{CONSIDERAÇÕES FINAIS}

Neste estudo foram encontrados ácaros em todas as amostras coletadas. Duas famílias, Glycyphagidae e Pyroglyphidae, se encontram entre a relação de famílias de ácaros mais recorrentes no Brasil. Assim como relatado em muitos trabalhos, as espécies mais recorrentes são Dermatophagoides ptronyssinus e Dermatophagoides farinae.

Das seis espécies identificadas, três são alergênicas e estão ligadas aos sintomas de doenças alérgicas respiratórias. D. ptreonyssinus, D. farinae e Blomia tropicalis apresentam alérgenos que em contanto com indivíduos sensibilizados são reconhecidos pela IgE e desencadeiam a resposta imunológica. Diante disso, reforça-se a importância do conhecimento da acarofauna em poeira domiciliar como medida adicional de prevenção de sintomas de doenças respiratórias como asma e rinite alérgica.

Duas das espécies encontradas, Dermatophagoides deanei e Sturnophagoides brasiliensis, são pouco descritas na literatura. Ainda não foi identificada a relação dessas espécies com o aparecimento de sintomas de doenças alérgicas respiratórias. Necessita-se de mais estudos sobre essas duas espécies para que essa relação seja verificada.

Incomum em poeira domiciliar a espécie parasita, Sarcoptes scabiei, também foi identificada nesse estudo. Essa informação reforça a importância dos cuidados necessários para o diagnóstico e tratamento de doenças em animais domésticos, principalmente, quando se trata de animais abandonados, que viviam na rua e foram adotados. Além disso, é imprescindível a higienização periódica dos locais da residência os quais esses animais possuem livre acesso.

Através desse estudo foi possível orientar os moradores das residências universitárias da cidade de Uberaba, onde as amostras foram coletadas, sobre os cuidados necessários para a higienização dos sofás e outros microambientes adequados para a nidificação e proliferação de ácaros. Entre os cuidados destacamse a utilização de capas em almofadas, sofás e colchões; lavagem periódica dessas capas e das cortinas presentes na residência e a retirada periódica da poeira de móveis e prateleiras.

\section{REFERÊNCIAS}

ABBAS, A.K; LICHTMAN, A.H; PILLAI, S. Imunologia básica: funções e distúrbios do sistema imunológico. 4. ed. Rio de Janeiro (RJ): Elsevier, 2014. xii, 320 p. 
ALMEIDA, K.C. Identificação de isoformas de Blomia tropicalis (Acari: Echimyopodidae) ligantes de anticorpos IgE de pacientes sensibilizados a ácaros de poeira domiciliar. 2010. $109 \mathrm{f}$. Tese (Doutorado em Imunologia e Parasitologia Aplicadas) - Universidade Federal do Triângulo Mineiro, Uberlândia, 2010.

BINOTTI, R.S.et al.House dust mites in Brazil - An annotated Bibliography. Memorial Instituto Oswaldo Cruz, Rio de Janeiro, v.96, n.8, p. 1177-1184, novembro, 2001.

BINOTTI, R.S. Levantamento da fauna acarina em amostras de poeira em domicílios da cidade de Campinas/SP. novembro, 2002. $121 \mathrm{f}$. Tese (Mestrado em Parasitologia) - Universidade Estadual de Campinas. Campinas, 2002.

BINOTTI, R.S.et al. Characterization of mites in sofa dust samples from homes in Campinas, Southeast Brazil. Revista Ciência Médica, Campinas, v.12, n.4, P. 327-330, outubro/dezembro, 2003.

CAMPOS, A.G.; LIPORACI, T.P.C.; TERRA, S.A. Identificação de ácaros da poeira domiciliar de Ituverava - SP. Nucleus, v. 7, n. 7, p. 169-179, abr. 2010. Disponível em: http://www.nucleus.feituverava.com.br/index.php/nucleus/article/view/332

COLLOFF, M.J. Dust Mites. Collingwood: CSIRO Publishing, 2009. 582 p.

ESTEVES; P.C., ROSARIO; N.A., Zavadniak A.F. Prevalence of perennial and seasonal rhinitis in Curitiba, Brazil. Allergy \& Clinical Immunology International, v. 18 (Supp2), p. 138, 2000.

FAIN, A. Deux nouvelles espèces de Dermatophagoides : Rattachement de cette sous-famille aux Pyroglyphidae (Sarcoptiformes). Acarologia. v.9, n.1, p.870-881, 1967.

FARIA, A.M. Tratamentos convencionais e fitoterápicos para controle de Sarna Sarcóptica nos animais domésticos (Revisão de literatura). Universidade Federal de Goiás, Goiânia, 2011.

FERNADEZ-CALDAS, E., PUERTA, L. MERCADO, D., LOCKEY, R.F., CARABALLO, L.R.. Mite fauna, Der p1, Der f1 and Blomia tropicalis allergen levels in a tropical environment, Clinical and Experimental Allergy. Oxford, v.23, n.4, p. 292-297, 1997.

FLECHTMANN, C.H.W. Ácaros de importância Médico Veterinária. São Paulo: Nobel, 1973. 192p.

GALLI, S.J.; LANTZ, C.S. Allergy. In: PAUL, W.E. Fundamental Imunology. 4.ed. Philadelphia: Lippincontt - Raven, 1999, p. 1127-1174.

GALVÃO, A.B.; GUITTON, N.; Ácaros em poeira domiciliar das capitais brasileiras e Ilha Fernando de Noronha. Memorial Instituto Oswaldo Cruz, Rio de Janeiro, v.81, n.4, p.417-430, outubro/dezembro, 1986a.

GALVÃO, A.B.; GUITTON, N.; Dermatophagoides deanei SP.N., nova espécie de ácaro piroglifídeo encontrado no Brasil, em poeira domiciliar. Memorial Instituto Oswaldo Cruz, Rio de Janeiro, v.81, n.2, p.241-244, abril/junho, 1986b.

GALVÃO, A.B.; GUITTON, N.; Noções de estrutura e biologia dos ácaros. Memorial Instituto Oswaldo Cruz, Rio de Janeiro, v.84, n.4, p.223-239, 1989.

JESUS, J.R. Investigação sobre associação entre ácaros da poeira, atopia, manifestações alérgicas e infecções intestinais helmínticas. 2010. 116 f. Tese (Doutorado em Imunologia) - Universidade Federal da Bahia, Salvador, 2010.

JOHANSSON, S.G.O.et al. Revised nomenclatre for allergy for global use: Reporto f the Nomenclature Review Committee os the World Allergy Organization, October 2003, J Allergy Clin Immunol, v.113, n.5, p. 832-836, 2004 
MIYAMOTO, T. Environmental impacto $n$ hypersensivity and allergy. In: JOHANSSON, S.G.O. (eds) Progress in allergy and clinical Imunology. v.3, Stockholm: Hogrefe \& Huber publishers. 1995, 480p., $81-82$

PANIGALLI, G.; GIROTTO, C. Ocorrência e diversidade de ácaros (Acari, Arachinida) no acervo e ambientes da Biblioteca Universitária Central da UNOESC Xanxerê. Unoesc \& Ciência - ACBS Edição especial, p. 103-112, 2014.

PIKE, A.J.; CUNNINGHAM, M.J.; LESTER, P.J. Development of Dermatophagoides pteronyssinus (Acari: Pyroglyphidae) at constant and simultaneously fluctuating temperature and humidity conditions. Journal of Medical Entomology, v.42, p. 266-269, 2005.

RODRIGUES, T.O.S. Sarna Humana. 2014. 66 f. Tese (Mestrado em Ciências Farmacêuticas) Universidade Fernando Pessoa, Porto, 2014.

SEGUNDO, G.R.S.et al. Diversity of allergen exposre: Implications for the efficacy of environmental control. Brazilian Journal of Otorhinolaryngology, v. 75, n. 2, p. 311-316, 2009.

SOUZA, C.C.T. Padrão de alérgenos inaláveis na poeira domiciliar em pacientes atópicos de Curitiba. 2013. 100 f. Tese (Mestrado em Pediatria) - Universidade Federal do Paraná, Curitiba, 2013.

SOUZA, C.C.T.; ROSARIO FILHO, N.A. Perfil de Aeroalérgenos intradomiciliares comuns no Brasil: revisão dos últimos 20 anos. Revista Brasileira de Alergia e Imunopatologia, São Paulo, v.35, n.2, 2012.

TERRA, S. A. et al. Mite allergen levels and acarologic analysis in house dust samples in Uberaba, Brazil. Journal of Investigational Allergology and Clinical Immunology.v.14, n.

3, p. 232-237, 2004. 